NDA-FP-33

May 1997

\title{
Trace Anomaly and Non-Local Effective Action for 2D Conformally Invariant Scalar Interacting with Dilaton
}

\author{
ShIN'ICHI NOJIRI and SERGei D. ODINTSOV \\ Department of Mathematics and Physics \\ National Defence Academy \\ Hashirimizu Yokosuka 239, JAPAN \\ A Dep.de Fisica \\ Universidad del Valle \\ AA25360, Cali, COLUMBIA \\ and \\ Tomsk Pedagogical Univer. \\ 634041 Tomsk, RUSSIA
}

\begin{abstract}
Using the results of the calculation of the one-loop effective action (E. Elizalde et al., Phys.Rev. D49 (1994) 2852), we find the trace anomaly for most general conformally invariant 2D dilaton coupled scalar-dilaton system (the contribution of dilaton itself is included). The non-local effective action induced by conformal anomaly for such system is found. That opens new possibilities in generalizing of CGHS-like model for the study of back reaction of matter to $2 \mathrm{D}$ black holes.
\end{abstract}

\footnotetext{
1 e-mail : nojiri@cc.nda.ac.jp

2 e-mail : odintsov@quantum.univalle.edu.co
} 
1. In the study of back reaction of quantum conformal matter to the evaporation of 2D black holes, the trace anomaly plays an important role. For example in the seminal works [5] and [6] working in the large- $N$ approximation (where $N$ is the number of scalars minimally coupled to dilaton), such trace anomaly has been used for the calculation of anomaly-induced effective action. This action represents back-reaction of matter to dilaton gravity theory and it may significally change the properties of $2 \mathrm{D}$ black holes and Hawking radiation [5, 6]. Moreover, such trace anomaly is directly responsible for Hawking radiation.

In more general dilaton gravity models (for such models, see [7]) where scalar non-minimally couples with dilaton the trace anomaly is more complicated. That is this trace anomaly which should be used in the generalizing of CGHS model where back-reaction is given by dilaton-coupled scalar. Few days ago, the calculation of trace anomaly for dilaton coupled scalar has been presented in ref. [3] (for a very specific choice of dilaton coupling). The purpose of the present note is to find the trace anomaly for most general conformally invariant dilaton-scalar system. Actually, such result is the byproduct of the calculation of the one-loop effective action for correspondent system which was done sometime ago in ref. [] $[$. We also find the anomalyinduced effective action for corespondent system.

2. Let us calculate trace anomaly for the system of scalar fields interacting with dilaton. In this calculation, we will take into account also the contribution of dilaton to trace anomaly.

The most general action to start with is given by

$$
S=\int d^{2} x \sqrt{g}\left\{-\frac{1}{2} Z(\phi) g^{\mu \nu} \partial_{\mu} \phi \partial_{\nu} \phi+\frac{1}{2} f(\phi) g^{\mu \nu} \partial_{\mu} \chi_{i} \partial_{\nu} \chi_{i}\right\}
$$

where $\phi$ is dilaton, $Z, f$ are the arbitrary dilaton functions, $\chi$ is scalar field, $i=1, \cdots, N$. Here dilaton and scalars are quantum fields and gravitational field is considered to be external field.

It is clear that such theory is conformally invariant in two dimensions. Hence, on classical level the trace of energy-momentum tensor is zero, while on quantum level, it is not zero (conformal or trace anomaly).

We work on the background with non-zero background dilaton and nonzero background scalar. The calculation of the one-loop effective action for such system has been done in all details, in ref.[1] (see Eq.(41)). The result 
looks as follows:

$$
\begin{aligned}
\Gamma_{\text {div }}= & -\frac{1}{4 \pi(n-2)} \int d^{2} x \sqrt{g}\left\{-\frac{N+1}{6} R+\left(\frac{f^{\prime 2}}{2 f Z}-\frac{f^{\prime \prime}}{2 Z}\right)\left(\nabla^{\lambda} \chi_{i}\right)\left(\nabla_{\lambda} \chi_{i}\right)\right. \\
& \left.+\left(\frac{N f^{\prime \prime}}{2 f}-\frac{N f^{\prime 2}}{4 f^{2}}-\frac{Z^{\prime 2}}{4 Z^{2}}\right)\left(\nabla^{\lambda} \phi\right)\left(\nabla_{\lambda} \phi\right)+\left(\frac{N f^{\prime}}{2 f}-\frac{Z^{\prime}}{2 Z}\right) \Delta \phi\right\}
\end{aligned}
$$

Here $\phi, \chi_{i}$ denote the dilaton and scalar background correspondently.

It is widely well-known that one can easily get the trace anomaly of conformally invariant system just by calculating the corresponding divergent effective action multiplied to $(n-2)$ in 2 dimensions (in other words, as coefficient of the pole). Hence, the trace anomaly of the system under discussion is given by

$$
\begin{aligned}
T= & \frac{1}{24 \pi}\left\{(N+1) R-3\left(\frac{f^{\prime 2}}{f Z}-\frac{f^{\prime \prime}}{Z}\right)\left(\nabla^{\lambda} \chi_{i}\right)\left(\nabla_{\lambda} \chi_{i}\right)\right. \\
& \left.-3\left(\frac{N f^{\prime \prime}}{f}-\frac{N f^{\prime 2}}{2 f^{2}}-\frac{Z^{\prime 2}}{2 Z^{2}}\right)\left(\nabla^{\lambda} \phi\right)\left(\nabla_{\lambda} \phi\right)-3\left(\frac{N f^{\prime}}{f}-\frac{Z^{\prime}}{Z}\right) \Delta \phi\right\}
\end{aligned}
$$

For purely scalar system coupled with dilaton the trace anomaly looks like:

$$
\begin{aligned}
T= & \frac{1}{24 \pi}\left\{N R-3 N\left(\frac{f^{\prime \prime}}{f}-\frac{f^{2}}{2 f^{2}}\right)\left(\nabla^{\lambda} \phi\right)\left(\nabla_{\lambda} \phi\right)\right. \\
& \left.-3 \frac{N f^{\prime}}{f} \Delta \phi\right\}
\end{aligned}
$$

Hence, we got the trace anomaly for scalar coupled with dilaton and for dilaton itself as by-product of the calculation of the effective action in ref. [1] .

Let us consider very special case $N=1$ (single scalar) and $f(\phi)=\mathrm{e}^{-2 \phi}$. Then

$$
T=\frac{1}{24 \pi}\left\{R-6\left(\nabla^{\lambda} \phi\right)\left(\nabla_{\lambda} \phi\right)+6 \Delta \phi\right\}
$$

The calculation of conformal anomaly has been done recently in the last case, using zeta-regularization method (see [2] for a review) in the ref.[3]. Comparing our expression (5) with corresponding expression of ref. [3], we see the difference in coefficient of the last term (total divergence) in Eq.(5), which is denoted by $q_{3}$ in ref.[3]. The disagreement is presumably caused by the fact that in getting of expression (2), one could drop some total divergence 
term as we are only interesting in the dilaton dependence of trace anomaly. In fact, the last term in eq.(3.3) in ref.[3] is, as written, is not unique and the term can be

$$
q_{3}\left\{a \phi R+(1-a) \square \phi \frac{1}{\square} R\right\}
$$

or more general form. Here $a$ is an arbitrary or undetermined constant. The authors of ref. [3] claimed that the difference is total derivative and can be neglected but it would not be true. As in section 3.1 in ref. [3], if we consider the case where $\phi$ is a constant $\phi=\phi_{0}$, we obtain

$$
q_{3} a \phi_{0} R
$$

instead of the last term in eq.(3.4) of ref.[3]. This tells $q_{3}$ cannot be determined due to the undetermined constant $a$. It indicates that the coefficient of total divergence term in the trace can not be derived in unique way. The coefficients of first two terms in (5) coincide with the calculation of ref. [3].

Thus we got the trace anomaly for most general 2D dilaton coupled scalardilaton system with arbitrary dilaton interactions.

3. Using the trace anomaly obtained in the previous section (see eq.(3), we may calculate the non-local effective action induced by trace anomaly.

Let us write the general form of trace anomaly as following

$$
\begin{aligned}
T= & c R+F_{1}(\phi)\left(\nabla^{\lambda} \chi_{i}\right)\left(\nabla_{\lambda} \chi_{i}\right)+F_{2}(\phi)\left(\nabla^{\lambda} \phi\right)\left(\nabla_{\lambda} \phi\right) \\
& +F_{3}(\phi) \Delta \phi
\end{aligned}
$$

where the explicit form of $c, F_{1}, F_{2}, F_{3}$ is evident from the comparison with (5):

$$
\begin{aligned}
& c=\frac{N+1}{24 \pi}, \quad F_{1}(\phi)=-\frac{1}{8 \pi}\left(\frac{f^{\prime 2}}{f Z}-\frac{f^{\prime \prime}}{Z}\right), \\
& F_{2}(\phi)=-\frac{1}{8 \pi}\left(\frac{N f^{\prime \prime}}{f}-\frac{N f^{\prime 2}}{2 f^{2}}-\frac{Z^{\prime 2}}{2 Z^{2}}\right), \\
& F_{3}(\phi)=-\frac{1}{8 \pi}\left(\frac{N f^{\prime}}{f}-\frac{Z^{\prime}}{Z}\right) .
\end{aligned}
$$

The (non-local) effective action induced by the conformal anomaly is defined as

$$
\int d^{2} x \sqrt{g} T=\left.2 \frac{d W}{d t}\right|_{t=1}
$$


where $\tilde{g}^{\mu \nu}=t^{-1} g_{\mu \nu}$. In other words, the effective action (10) should derive the anomaly $T$ (see 画).

First of all, let us rewrite the trace anomaly in the different form. To do this, first of all, we introduce the function $\tilde{F}_{3}(\phi)$ such that $\frac{\partial \tilde{F}_{3}(\phi)}{\partial \phi}=F_{3}(\phi)$. Then $\tilde{F}_{3}(\phi)=\int F_{3}(\phi) d \phi$ and

$$
\begin{aligned}
T= & c R+F_{1}(\phi)\left(\nabla^{\lambda} \chi_{i}\right)\left(\nabla_{\lambda} \chi_{i}\right) \\
& +\left(F_{2}(\phi)-\frac{\partial F_{3}(\phi)}{\partial \phi}\right)\left(\nabla^{\lambda} \phi\right)\left(\nabla_{\lambda} \phi\right)+\Delta \tilde{F}_{3}(\phi)
\end{aligned}
$$

At the next step, we introduce the function $\tilde{F}_{2}(\phi)$ where $\frac{\partial \tilde{F}_{2}(\phi)}{\partial \phi}=$ $\left(F_{2}(\phi)-\frac{\partial F_{3}(\phi)}{\partial \phi}\right)^{\frac{1}{2}}$. Then

$$
\begin{aligned}
T= & c R+\left(\nabla^{\lambda} \tilde{\chi}_{i}\right)\left(\nabla_{\lambda} \tilde{\chi}_{i}\right) \\
& +\nabla^{\lambda} \tilde{F}_{2}(\phi) \nabla_{\lambda} \tilde{F}_{2}(\phi)+\Delta \tilde{F}_{3}(\phi)
\end{aligned}
$$

where $\nabla^{\lambda} \tilde{\chi}_{1} \equiv F_{1}^{\frac{1}{2}}(\phi) \nabla^{\lambda} \chi_{i}$

The non-local effective action induced by the trace anomaly of such form has been already obtained in ref.[3]. Applying this result to Eq.(12) we get

$$
\begin{aligned}
W= & -\frac{1}{2} \int d^{2} x \sqrt{g}\left[\frac{c}{2} R \frac{1}{\Delta} R+\left(\nabla^{\lambda} \tilde{\chi}_{i}\right)\left(\nabla_{\lambda} \tilde{\chi}_{i}\right) \frac{1}{\Delta} R\right. \\
& +\nabla^{\lambda} \tilde{F}_{2}(\phi) \nabla_{\lambda} \tilde{F}_{2}(\phi) \frac{1}{\Delta} R+\tilde{F}_{3}(\phi) R
\end{aligned}
$$

One can rewrite this efective action in terms of original functions;

$$
\begin{aligned}
W= & -\frac{1}{2} \int d^{2} x \sqrt{g}\left[\frac{c}{2} R \frac{1}{\Delta} R+F_{1}(\phi)\left(\nabla^{\lambda} \chi_{i}\right)\left(\nabla_{\lambda} \chi_{i}\right) \frac{1}{\Delta} R\right. \\
& \left.+\left(F_{2}(\phi)-\frac{\partial F_{3}(\phi)}{\partial \phi}\right) \nabla^{\lambda} \phi \nabla_{\lambda} \phi \frac{1}{\Delta} R+R \int F_{3}(\phi) d \phi\right]
\end{aligned}
$$

Thus, we got the trace anomaly induced action for most general 2D dilaton coupled scalar-dilaton system. This action should be added to the classical action of dilaton gravity interactng with above system in order to study the properties of black holes and Hawking radiation with account of backreaction. We hope to return to the study of applications of the effective action (114) in the evaporation of $2 \mathrm{D}$ black holes taking into account quantum effects in near future. 


\section{References}

[1] E. Elizalde, S. Naftulin and S.D. Odintsov, Phys. Rev. D49 (1994) 2852.

[2] E. Elizalde, S.D. Odintsov, A. Romeo, A.A. Bytsenko and S, Zerbini, Zeta-regularization technique with applications, World Sci., Singapore, 1994.

[3] R. Bousso and S.W. Hawking, preprint hep-th/9705236.

[4] S.D. Odintsov and I.L. Shapiro, Class. Quant. Grav. 8 (1991) L57.

[5] C.G. Callan, S.B. Giddings, J.A. Harvey and A. Strominger, Phys. Rev. D45 (1992) 1005.

[6] J.G. Russo, L. Susskind and L. Thorlacius, Phys. Lett. B292 (1992) 13.

[7] S.D. Odintsov and I.L. Shapiro, Phys.Lett. B263 (1991) 183; T. Banks and M. O'Loughlin, Nucl.Phys. B362 (1991) 649. 